\title{
The party politics of the Euro crisis in the German Bundestag: Frames, positions and salience
}

\author{
Arndt Wonka \\ Bremen International Graduate School of Social Sciences (BIGSSS) \\ University of Bremen \\ Postfach 330440 \\ D - 28334 Bremen \\ Germany \\ awonka@bigsss.uni-bremen.de
}


Online Appendix „The party politics of the Euro crisis in the German Bundestag: Frames, salience and conflict" [to be published on author's homepage]

\section{Appendix A1: Identification of "Bundestag" debates and newspaper articles}

The following keywords were used to identify parliamentary discussions on the respective instruments:

Fiscal Treaty: Fiskalpakt, Fiskalvertrag, Fiskal-

ESM: ESM, Stabilisierungsmechanismus, Stabilitätsmechanismus

EFSF: EFSF, fazilität

Sixpack: Sixpack, Six Pack, Stabilitätspakt, makroökonomische Ungleichgewichte

In addition, more general keywords were used to make sure that relevant parliamentary discussions were not missed in which the instruments were discussed without, however, referring to their technical names:

General keywords: EU, Krise, europäisch, Finanzstabilität, Rettungsschirm

To identify the news coverage of parliamentary debates, the newspapers' online archives were searched with similar keywords. Since, however, newspaper search was restricted to one day before and two days after the debate, the respective editions of the papers could, in addition, be completely scrutinized to identify relevant articles. To identify the TV coverage of the discussions I relied on livestreams of the Tagesschan of the day on which the respective parliamentary discussion was taking place. Each 15 minutes broadcast of the Tagesschau of the respective day was analyzed for this purpose.

\section{Appendix A2: Coding}

In the four parliamentary debates analyzed in this paper, parliamentarians do address a number of issues which are only indirectly related to the EFSF, the Sixpack, the ESM and the Fiscal Treaty. For the analysis presented in the paper, however, only those statements were considered and coded which explicitly dealt with the respective instrument. To identify these statements and the frames which they express, I relied on the protocol of the respective debate. In a first round, the coder went through the complete protocol and identified the respective frames on the basis of the categories listed in Table 1 of the paper. During this first step, the author discussed individual framing decisions with the coder. After this first step was completed, the categories and their definition were discussed on the basis of the first round of coding. This led to a further specification and additional definitional re-calibration of the codes, which the author discussed with the coder. In a second round, the coder went back to the protocols and the results of the first round of coding to check whether, on the basis of the refined codebook, additional statements and frames could be identified in the original protocols and to check the coding results of the first round against the more fully specified codebook. 


\section{Coding scheme}

\begin{tabular}{|c|c|c|}
\hline CATEGORY & DEFINITION & EXAMPLE FROM PARLIAMENTARY DEBATES \\
\hline $\begin{array}{l}\text { Economic } \\
\text { Growth }\end{array}$ & $\begin{array}{l}\text { Effects of crisis instrument on economic growth; } \\
\text { alternative/complementary measures which } \\
\text { stimulates growth }\end{array}$ & $\begin{array}{l}\text { Investitionen sind notwendig, um wirtschaftliches W achstum zu erzengen. Deshalb haben } \\
\text { wir Gott sei Dank diesen Merkel'schen Weg in den Verbandlungen korrigiert }\end{array}$ \\
\hline $\begin{array}{l}\text { Economic } \\
\text { Employment }\end{array}$ & $\begin{array}{l}\text { Effects of a crisis instrument on employment; } \\
\text { effects of employment situation on } \\
\text { formulation/content of crisis measures }\end{array}$ & $\begin{array}{l}\text { Deshalb muss es im Kern immer wieder um die Frage gehen: Wie können wir die } \\
\text { Beschäftigung steigern? Insofern begrüßen wir den Ansatz der Kommission, die jetzt } \\
\text { vorgeschlagen hat, insbesondere die steuerliche Belastung von Arbeit zu reduzieren }\end{array}$ \\
\hline $\begin{array}{l}\text { Economic } \\
\text { Fiscal stability }\end{array}$ & $\begin{array}{l}\text { Effects of crisis instrument on countries' budgetary } \\
\text { situation; fiscal situation and fiscal policy-making } \\
\text { more generally (policies which affect state income } \\
\text { vs. state expenditures) }\end{array}$ & $\begin{array}{l}\text { Heute wurde bereits mehrfach zum Ausdruck gebracht, dass beides, ESM und Fiskalpakt, } \\
\text { notwendige Voraussetzungen sind, um eine Stabilitätskultur in Europa weiter aufzubauen }\end{array}$ \\
\hline $\begin{array}{l}\text { Economic } \\
\text { Currency Union }\end{array}$ & $\begin{array}{l}\text { General viability of Euro; economic/political } \\
\text { effects of Euro; assessment of size/members of } \\
\text { Euro zone }\end{array}$ & $\begin{array}{l}\text { Wir Deutschen hatten in den letzten Jahren Exporte in Höhe von } 800 \text { bis } 900 \\
\text { Milliarden Euro zu verzeichnen; davon gingen rund zwei Drittel in den Bereich der } \\
\text { Europäschen Union, davon wiederum der überwiegende Anteil in den Bereich der Euro- } \\
\text { Zone }\end{array}$ \\
\hline $\begin{array}{l}\text { Economic } \\
\text { EU redistribution }\end{array}$ & $\begin{array}{l}\text { Monetary transfers across member states (via EU } \\
\text { subsidies and/or credits), organized by EU/crisis } \\
\text { instruments; redistributive effects and concrete } \\
\text { beneficiaries of redistribution (banks, capitalists, } \\
\text { firms, citizens, the poor...) }\end{array}$ & $\begin{array}{l}\text { Wer von dieser Umverteilung tatsächlich profitiert, kann man in Griechenland deutlich } \\
\text { sehen. Zu Beginn seiner vermeintlichen Rettung hatte Griechenland } 300 \text { Milliarden Euro } \\
\text { Schulden, die von Banken, Hedgefonds und vermögenden Privatanlegern gehalten wurden. } \\
\text { Heute hat Griechenland } 360 \text { Milliarden Euro Schulden, aber für } 300 \text { Milliarden davon } \\
\text { haften jetzt die europäischen Steuerzabler }\end{array}$ \\
\hline $\begin{array}{l}\text { Cultural } \\
\text { National identity }\end{array}$ & $\begin{array}{l}\text { Reference to characteristics of the Germans (or } \\
\text { another people/country) which are relevant for } \\
\text { crisis; the rights and the destiny of "the Germans" } \\
\text { or Germany (not the government) }\end{array}$ & $\begin{array}{l}\text { Jeder muss wissen, dass auch in Zukunft die nationale Identität nicht in Europa aufgehen } \\
\text { darf. Deswegen muss das auch für die Wirtschafts- und Währungsunion gelten }\end{array}$ \\
\hline $\begin{array}{l}\text { Cultural } \\
\text { European Identity }\end{array}$ & $\begin{array}{l}\text { Reference to collective European/ EU values } \\
\text { and/or characteristics; shared "fate" of all } \\
\text { Europeans in the crisis, which asks for action; } \\
\text { effects of crisis measure on the formation of an } \\
\text { European identity }\end{array}$ & Was haben Sie denn dagegen, festzustellen, dass wir Teil der europäischen Familie sind? \\
\hline $\begin{array}{l}\text { Cultural } \\
\text { National stereotypes }\end{array}$ & $\begin{array}{l}\text { Attribution of specific characteristics of a } \\
\text { people/country which had an effect on crisis } \\
\text { dynamics; the characteristics of "the } \\
\text { Germans/Germany" vs. the characteristics of } \\
\text { "the/an-other"; German political/ "policy" culture }\end{array}$ & $\begin{array}{l}\text { Ich möchte, dass unserer Bevölkerung eine Frage beantwortet wird: Sind Länder wie } \\
\text { Griechenland, Irland und Portugal in einer Krise, weil die Leute dort faul und raffgierig sind } \\
\text { - so lautet eine immer wieder anklingende rassistische Antwort-, oder hat das, wie wir } \\
\text { meinen, ganz, andere Ursachen? Darüber muss aufgeklärt werden }\end{array}$ \\
\hline
\end{tabular}




\begin{tabular}{|c|c|c|}
\hline $\begin{array}{l}\text { Cultural } \\
\text { (Im-) Migration }\end{array}$ & $\begin{array}{l}\text { Effects of crisis measures on increase/reduction of } \\
\text { migration of citizens between member states; } \\
\text { migration as a general consequence of Euro crisis; } \\
\text { multiculturalism and cultural heterogeneity in EU } \\
\text { member states }\end{array}$ & NO FRAME \\
\hline $\begin{array}{l}\text { Cultural } \\
\text { Sovereignty }\end{array}$ & $\begin{array}{l}\text { Effects of crisis instrument on national } \\
\text { governments'/ country's capacity to act } \\
\text { independently in policy-making; a people's capacity } \\
\text { to "steer" its own "destiny" }\end{array}$ & $\begin{array}{l}\text { Ja, ich weiß sehr genau, dass wir damit die Tür für den Verlust von Souveränität öffnen. Ja, } \\
\text { ich weiß sehr genau, dass wir alle an diesem Europa in seiner Unvollkommenheit hin und } \\
\text { wieder verzweifeln. Trotzdem werde ich für den Fiskalpaket und für den ESM stimmen }\end{array}$ \\
\hline $\begin{array}{l}\text { Cultural } \\
\text { Solidarity }\end{array}$ & $\begin{array}{l}\text { Need/wish to help citizens of other member states } \\
\text { in the EU very broadly and/or as an ideal (as } \\
\text { opposed to concrete payments which are covered } \\
\text { by re-distributive dimension) }\end{array}$ & Wir sollten den portugiesischen Bürgerinnen und Bürgern diese Chance nicht verwehren \\
\hline $\begin{array}{l}\text { Institutional } \\
\text { EU centralization/ integration }\end{array}$ & $\begin{array}{l}\text { Transfer of competencies to EU level and/or } \\
\text { specific EU institutions through crisis measure; call } \\
\text { for (de-) centralized institutional solutions; effect of } \\
\text { crisis instrument on relative power of national and } \\
\text { EU level/actors }\end{array}$ & $\begin{array}{l}\text { Wenn diese Verpflichtungen in Vereinbarungen festgelegt werden und wenn sie - das muss } \\
\text { Bestandteil der Regelung sein - durch die europäischen Institutionen, so wie es der Vertrag } \\
\text { vorsieht, im Rabmen eines strengen Monitorings überwacht werden, [...] }\end{array}$ \\
\hline $\begin{array}{l}\text { Institutional } \\
\text { Effectiveness of instruments }\end{array}$ & $\begin{array}{l}\text { Instruments (in-) appropriateness to reach the } \\
\text { intended effect; }\end{array}$ & $\begin{array}{l}\text { Die harten Bedingungen des Rettungsschirms erfüllen ibre Funktion. Es gibt keine Anreize, } \\
\text { die Staatsverschuldung weiter in die Höhe zu treiben }\end{array}$ \\
\hline $\begin{array}{l}\text { Institutional } \\
\text { Democratic control } \\
\text { / accountability }\end{array}$ & $\begin{array}{l}\text { Effects of crisis measure on democratic } \\
\text { accountability and control; effects of measure on } \\
\text { chain of delegation (voter -> parliament -> } \\
\text { government }->\text { policy); call for empowerment of } \\
\text { parliament (national or EP) to increase democratic } \\
\text { control over content/application of measure }\end{array}$ & $\begin{array}{l}\text { Ich glaube, wir sind gut beraten, wenn wir das, was als Verfassungsänderung nötig oder } \\
\text { gegebenenfalls als Volksentscheid erforderlich ist, hier im Parlament bzw. in Deutschland } \\
\text { regeln und uns nicht auf dem Umweg über europä̈sche Verträge Änderungen unserer } \\
\text { Verfassung sozusagen beibringen lassen }\end{array}$ \\
\hline
\end{tabular}




\section{Appendix A3: Correlation Bundestag debates and TV and newspaper coverage}

To explore the relationship between the scope of parliamentary debate and public reach, i.e. the scope of media coverage, I relied, in addition to Graph 2, on the plots below.
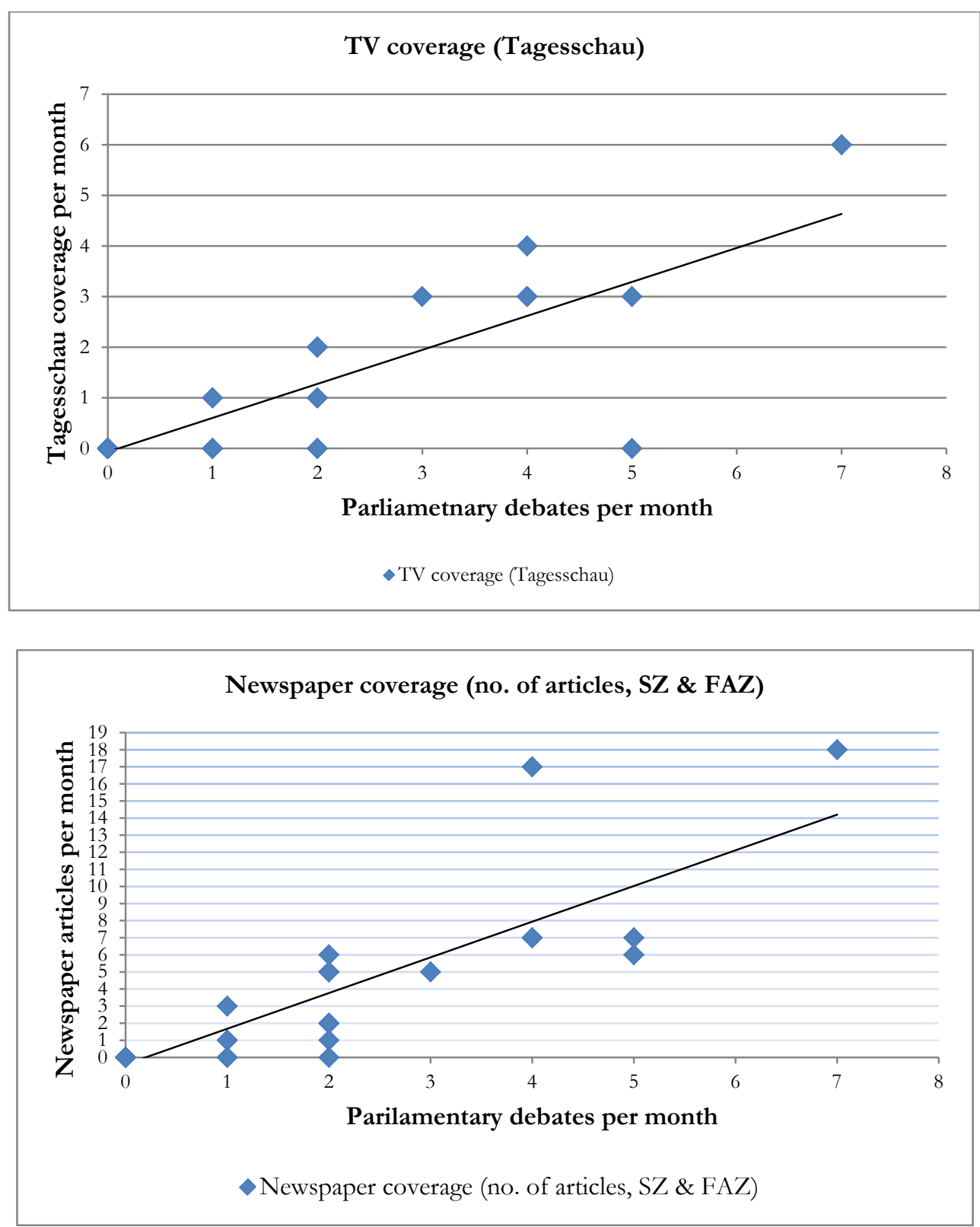